tels Kenntnisstandprüfung nachweisen mussten, wenn sie nach dem Erhalt der Staatsbürgerschaft eine Approbation beantragten. Dass die Klägerin diese Prüfung zweimal nicht bestanden hatte, wurde als nicht relevant erachtet. Auch der Hinweis, dass die Zentralstelle für ausländisches Bildungswesen, die als Gutachterstelle für Anerkennungsstellen wirkt, die russische Ausbildung nicht als objektiv gleichwertig ansieht und regelmäßig auf kürzere Schul- und Praktikazeiten verweist, wurde zurückgewiesen, da die über zwanzigjährige Berufserfahrung keine Rolle bei der Bewertung gespielt hatte. Der individuelle Kenntnisstand sei grundsätzlich zu prüfen, daher wurde auch dem Einwand bezüglich genereller bisheriger Erfahrungen der Anerkennungspraxis keine Bedeutung zugemessen, da er nichts über den jeweiligen Einzelfall aussage.

Obwohl das Gericht keine Diskriminierung der Klägerin in der Ungleichbehandlung gegenüber baltischen Staatsangehörigen sah, die eine automatische Anerkennung ihrer sowjetischen Arztausbildungen erlangen können, wurde doch auf spezifische Auswirkungen der Richtlinie 2005/36/EG hingewiesen. Demnach habe der deutsche Gesetzgeber keine Gefahr für die Bevölkerung erkennen können, als er einer Anerkennung baltischer Staatsangehöriger zustimmte; demnach sei ein gleichwertiger Kenntnisstand mit sowjetischen Ausbildungen durchaus möglich.

Es bleibt abzuwarten, welche Auswirkungen dieses Urteil für die deutsche Anerkennungspraxis haben wird. Dass ausländischen Ärzt(inn)en nach Erhalt der Staatsangehörigkeit nun eine Bewertung ihrer Berufserfahrung nicht mehr verweigert werden kann, um die Gleichwertigkeit festzustellen, könnte sich auch auf andere Berufe auswirken.

Kompetenzbasierte Anerkennungsverfahren als neuen Standard einzuführen, würde sich nicht nur integrationspolitisch positiv auswirken. Auch bildungspolitisch ist die Wertschätzung individuell feststellbarer Kompetenzen gegenüber der formalen Qualifikation, die vielleicht schon Jahre zurückliegt und nichts über tatsächlich vorliegende Kenntnisse und Fähigkeiten aussagt, im Fokus der europäischen Diskussionen um Modernisierungen im Bildungssystem. Der Europäische Qualifikationsrahmen, der derzeit erstellt wird, könnte auch im Bereich der Anerkennung von ausländischen Abschlüssen zu weiteren Reformen führen.

\title{
Arbeitsmarktzugang und Ausbildungsbeihilfen
}

\author{
Prof. Dr. Dorothee Frings \\ Hochschule Niederrhein, Mönchengladbach
}

\section{Patriarchalische Struktur des Ausländerrechts}

Migration wird traditionell als männliches Phänomen wahrgenommen - so bleibt auch das deutsche Ausländerrecht weiterhin auf den männlichen Arbeitnehmer fokussiert.

Wenn das Bild der migrantischen Frau in unserer Gesellschaft noch immer geprägt ist vom Stereotyp der Kopftuch tragenden muslimischen Hausfrau oder der fast sprachlosen Putzfrau, die abends ihren Putzwagen durch leere Büroflure schiebt, so liegt die Verantwortung nicht allein bei psychologisch begründeten Zuschreibungen und Medienbildern, sondern auch bei einer Ausländerpolitik, die Frauen als Anhängsel ihrer Ehemänner behandelt hat.

- Das Aufenthaltsrecht knüpft an der Sicherung des Lebensunterhalts und damit an der Erwerbstätigkeit an; andere gesellschaftliche Beiträge - wie die Kindererziehung und Pflege von Angehörigen - werden ausgeblendet. Sie können bis heute allenfalls als Härtegesichtspunkte in besonders gelagerten Ausnahmefällen berücksichtigt werden; in der Regel nur, wenn es zur Vermeidung von Grundrechtsbeeinträchtigungen unabweisbar ist.

- Bis 2004 wurde nachziehenden Ehefrauen in den entscheidenden ersten Jahren ihres Aufenthalts der Zugang zum Arbeitsmarkt versperrt und ihnen so gesetzlich die Hausfrauenrolle verordnet.
- Familienbezogene Sozialleistungen galten bis vor wenigen Jahren als öffentliche Leistungen, die der Erteilung und Verlängerung der Aufenthaltserlaubnis entgegenstanden. Bis heute wird das Wohngeld als eine aufenthaltsschädliche Sozialleistung behandelt. Kinder zu haben wird immer noch zum Risiko des Aufenthaltsverlustes.

- Durch die Nichtanerkennung der ausländischen Qualifikationen werden Migrantinnen als „naturbegabt“ für die am schlechtesten bezahlten Bereiche personaler Dienstleistungen bewertet und in den prekären Sektor des Arbeitsmarktes abgedrängt.

Der erste Ausländerbeauftrage der Bundesregierung, Heinz Kühn, kritisierte in seinem berühmten Memorandum bereits vor genau 30 Jahren die Vernachlässigung der Arbeitsmarktintegration von Migrantinnen: „Mit gleicher Intensität müssen Möglichkeiten zur berufsbegleitenden Höherqualifizierung der ungelernten Beschäftigung, insbesondere auch der vielfach besonders benachteiligten ausländischen Mädchen ausgeschöpft werden. “1

Kühn kritisierte auch die Arbeitsmarktprüfung, nach der die Arbeitserlaubnis nur erteilt werden darf, wenn für den konkreten Arbeitsplatz keine sonstigen Arbeitsuchenden ver-

1 Kühn, Heinz, Stand und Weiterentwicklung der Integration der ausländischen Arbeitnehmer und ihrer Familien in der Bundesrepublik Deutschland, 6.5, Kühn-Memorandum, September 1979, erhältlich im Internet: <http://www.migration-online.de/data/ khnmemorandum_1.pdf $>$ (besucht am 12. Oktober 2009). 
Asylbewerberinnen:

1. Jahr Arbeitsverbot;

ab 2. Jahr Beschäftigungserlaubnis nur nachrangig

Geduldete:

1. Jahr Arbeitsverbot;

ab 2. Jahr Beschäftigungserlaubnis nur nachrangig;

nach vier Jahren oder zur Aufnahme einer Ausbildung ohne Arbeitsmarktprüfung; unbegrenztes Arbeitsverbot, wenn zum Zweck des Sozialleistungsbezugs eingereist oder das Abschiebehindernis selbst verschuldet wurde.

Studentinnen:

90 ganze oder 180 halbe Tage ohne Genehmigung;

selbständige Tätigkeit nur nach Ermessen

Alle sonstigen Ausländerinnen mit Aufenthaltserlaubnis, ausgenommen Studentinnen

Beschäftigungserlaubnis Anfangs nur nachrangig, nach drei Jahren Aufenthalt ohne Arbeits-

marktprüfung; selbständige Tätigkeit nur nach Ermessen.

Neu-EU:

Erstmalige Arbeitserlaubnis-EU nur nachrangig; selbständige Tätigkeit genehmigungsfrei

Alt-EU +

Drittstaatsangehörige mit Erwerbserlaubnis:

- Niederlassungserlaubnis

Genehmigungsfreie Ausübung jeder Form der Erwerbstätigkeit

- Anerkannte Flüchtlinge

- Familienangehörige von Deutschen

- Ehefrauen von Privilegierten

- Eigenständiger Aufenthalt nach Trennung

fügbar sind. Durch dieses Verfahren werde die Bildungsmotivation junger Menschen ausbremst und Begabungspotentiale verschenkt. $^{2}$

Dass ein Teil der Vorschläge 25 Jahre später ins Zuwanderungsgesetz aufgenommen wurde, ist nicht zuletzt einer intensiven Lobbyarbeit der verschiedenen Frauenverbände zu danken.

\section{Zugang zum Arbeitsmarkt für Migrantinnen}

Eine der wichtigsten Reformen in dem seit 2005 geltenden Aufenthaltsgesetz (AufenthG) ist die Regelung der Erwerbserlaubnis, die mit bestimmten Aufenthaltstiteln verbunden wird. ${ }^{3}$ Nachziehenden Ehefrauen wurden dadurch erstmals weitgehende Zugangsmöglichkeiten zur Erwerbstätigkeit eingeräumt. $^{4}$

Es folgte 2007 die uneingeschränkte Zulassung zur Erwerbstätigkeit im Rahmen der Probeaufenthaltserlaubnis nach $\mathbb{S} 104$ a AufenthG für langjährig Geduldete und 2009 die unbeschränkte Beschäftigungserlaubnis für Geduldete nach vier Jahren Aufenthalt oder für die Aufnahme einer Ausbildung.

Hinzu kommt eine begrenzte Öffnung der Grenzen für Akademikerinnen.

Mit den Neuregelungen sind die formalen Hürden für eine Erwerbstätigkeit von Frauen deutlich gesenkt worden. Da aber die Grundkonstruktion eines hierarchisch strukturierten
Arbeitsmarktzugangs nicht aufgegeben wurde, bleiben immer noch Frauen von Ausbildung und Arbeit ausgeschlossen.

Die praktischen Probleme für Frauen ohne deutsche Staatsangehörigkeit möchte ich an einigen Beispielen erläutern:

- Neu-Unionsbürgerinnen erhalten für die erste Arbeitsaufnahme nur nachrangig eine Arbeitserlaubnis-EU: ${ }^{5}$

Eine rumänische Staatsangehörige kommt nach Abbruch ihres Studiums der Elektrotechnik in Sofia nach Deutschland. Hintergrund ist - wie so oft - ein Mix von wirtschaftlichen und persönlichen Problemen, u.a. muss auch die Krankenbehandlung des Bruders finanziert werden. Als Unionsbürgerin hat sie freien Zutritt zum Bundesgebiet, sie kann sich zum Zwecke der Arbeitssuche auch ohne zeitliche Begrenzung hier aufhalten. Allerdings hat sie keine Chance eine Arbeit aufzunehmen, da sie als Neu-Unionsbürgerin eine Arbeitserlaubnis-EU nach $\mathbb{2} 284$ SGB III benötigt. Diese darf ihr nur für eine konkrete Stelle erteilt werden, für die keine Deutschen oder Unionsbürger am Arbeitsmarkt verfügbar sind. Da sie in der Kategorie „ungelernt" geführt wird, kann die Arbeitsagentur auf die Zahl der registrierten ungelernten Arbeitskräfte verweisen, die in ihrem Bezirk gemeldet sind, ohne eine konkrete Ver-

\footnotetext{
2 Ebd., 6.6, $35 \mathrm{f}$.

3 Z.B. $\$ 28$ Abs. 5 AufenthG.

4 Frings, Dorothee, Streit 2005, Heft 4, Beilage, VI.

$5 \S 284$ SGB III.
} 
fügbarkeitsprüfung hinsichtlich des gewünschten Arbeitsplatzes durchführen zu müssen.

Wie geht es weiter? Die Ausweichmöglichkeiten sind vielfältig. Sie macht sich als Anbieterin haushaltsnaher Dienstleistungen selbständig. Sie bittet eine Freundin, in Rumänien eine Firma anzumelden, lässt sich von ihr anstellen und als Dienstleistungsanbieterin nach Deutschland entsenden, zum Beispiel als Haushaltshilfe für Pflegebedürftige. Sie heiratet oder bekommt ein deutsches Kind. Sie übt drei Jahre lang Schwarzarbeiten im Haushalt aus, um durch Zeitablauf den Anspruch auf die Arbeitsberechtigung-EU zu erwerben. Was sie aber nicht tun kann, ist Leistungen nach SGB II beziehen, ${ }^{6}$ eine Qualifizierungsmaßnahme absolvieren (fehlende Integrationschancen) oder BAföG zur Weiterführung ihres Studiums beziehen. ${ }^{7}$

- Die Ehefrauen von Selbständigen und Ehemännern, die zum Zweck der Beschäftigung nach Deutschland gekommen sind, haben ebenfalls nur einen nachrangigen Zugang zum Arbeitsmarkt: ${ }^{8}$

Dem russischen Unternehmer wird eine Aufenthaltserlaubnis zur Gründung eines Handelsunternehmens nach $\mathbb{S} 21$ AufenthG erteilt. Seine Frau folgt ihm nach Deutschland. Sie hat bisher in Russland als Lehrerin gearbeitet. Sie hat keine rechtliche Möglichkeit, eine Anerkennung für ihren Abschluss zu erhalten. Ganz bescheiden sucht sie sich nun eine Tätigkeit als Verkaufshilfe bei einem Discounter, um zumindest unter Menschen zu kommen und so ihre deutschen Sprachkenntnisse zu verbessern. Aber auch hier gibt es keine Chance. Sie hat nach $\mathbb{S} 29$ Abs. 5 AufenthG erst nach zwei Jahren Eheleben in Deutschland einen Anspruch auf eine Erwerbserlaubnis, weil auch ihr Ehemann keine Erwerbserlaubnis besitzt, sondern lediglich eine Erlaubnis zur selbständigen Tätigkeit. Eine Beschäftigungserlaubnis kann sie nur erhalten, wenn keine bevorrechtigten Arbeitnehmerinnen für die Stelle verfügbar sind. Das Interesse der Arbeitgeberin an der Einstellung spielt dabei keine Rolle. Auch ein Integrationskurs kommt für sie nicht in Frage, weil sie bereits über ein Sprachniveau B 1 verfügt, mehr aber im Rahmen dieser Kurse nicht angeboten werden darf. Möglich bleibt die Arbeitslosmeldung bei der Arbeitsagentur; Integrationsmaßnahmen für Personen ohne Arbeitslosengeldbezug sind nach SGB III nur nach Ermessen vorgesehen. Im Rahmen des Ermessens wird zu berücksichtigen sein, dass sie keine Aussicht auf eine Beschäftigungserlaubnis hat und damit ein Integrationserfolg nicht erwartet werden kann. Zumindest zwei Jahre wird sie auf die Rolle als Hausfrau zurückgeworfen, die in ihrem bisherigen Leben biografisch nicht vorgesehen war.

- Geduldete unterliegen einem unbefristeten Arbeitsverbot, wenn sie das Abschiebehindernis schuldhaft herbeigeführt haben. Das Verschulden der Eltern wird den Kindern zugerechnet, so dass auch ihnen Ausbildung und Arbeit untersagt ist: ${ }^{9}$

Die türkisch-libanesische Familie lebt seit 18 Jahren in Deutschland, die Tochter ist hier geboren und jetzt 17
Jahre alt. Nach einem guten Realschulabschluss möchte sie eine Ausbildung als Immobilienkauffrau beginnen. Ein Ausbildungsplatz ist schon gefunden. Die gesamte Zeit über wurden der Familie Duldungen auf der Grundlage einer angenommenen türkischen Volkszugehörigkeit und libanesischen Staatsangehörigkeit erteilt, weil in diesen Fällen in der Regel keine Pässe zu erlangen sind. Tatsächlich lag jedoch eine türkische Staatsangehörigkeit vor. Wer davon in der Familie wusste oder nicht, ist kaum zu ermitteln. Die Ausländerbehörde stellt jedoch fest, dass die Eltern durch ein Verschweigen ihrer Staatsangehörigkeit eine Beendigung des Aufenthalts schuldhaft vereitelt haben. Dieses Verschulden wird der Tochter zugerechnet, weil es während ihrer Minderjährigkeit erfolgte. ${ }^{10}$ Damit wird die Tochter einem Arbeitsverbot unterworfen, welches sie an Haus und Herd bindet.

- Asylbewerberinnen erhalten für das gesamte Verfahren unabhängig von der Dauer - nur nachrangig eine Beschäftigungserlaubnis: ${ }^{11}$

Die irakische Asylbewerberin hat am Pariser Konservatorium und in Bagdad Klavier und Gesang studiert. Sie hat zahlreiche Kontakte in Deutschland. Eine Musikschule würde sie gerne als Dozentin engagieren und sie hat Auftrittsangebote in mehreren Städten. Im ersten Jahr kommt keine dieser Aktivitäten in Betracht. ${ }^{12}$ Anschließend könnte sie einen Antrag auf eine Beschäftigungserlaubnis stellen. Der Ausgang der Prüfung, ob keine anderen vergleichbaren Arbeitskräfte verfügbar sind, ist ungewiss. Den Auftrittsangeboten kann sie jedoch nicht nachkommen; nicht so sehr weil es sich um eine selbständige Tätigkeit handelt, ${ }^{13}$ sondern weil sie hierfür keine Erlaubnis zum Verlassen des zugewiesenen Bezirks erhalten kann. ${ }^{14}$

Eine Flüchtlingsfrau mit einer Ausbildung als Ingenieurin hätte voraussichtlich während der gesamten Zeit des Asylverfahrens keine Chance zu arbeiten.

- Alle Drittstaatsangehörigen, die nicht über eine Erwerbserlaubnis verfügen, benötigen eine ausländerrechtliche Genehmigung für eine selbständige Tätigkeit. Diese wird nur nach Ermessen erteilt. ${ }^{15}$ Für Geduldete und Asylbewerberinnen ist sie ausgeschlossen:

Einer bosnischen Staatsangehörigen wurde in Hinblick auf das Personensorgerecht für ein Kind, dessen Vater mit Flüchtlingsstatus in Deutschland lebt, eine Aufenthaltserlaubnis nach $\mathbb{2} 25$ Abs. 5 AufenthG erteilt. Sie findet zunächst eine Anstellung, die aber nach zwei Jahren im Zuge der Wirtschaftskrise betriebsbedingt gekündigt wird. Angesichts der schlechten Aussichten am Arbeitsmarkt über-

\footnotetext{
6 Ausschlussklausel in $\S 7$ Abs. 1 Satz 2 Nr. 2 SGB II.

$7 \S 8$ BAföG.

$8 \oint \oint 18,21$ i.V.m. $\S 28$ Abs. 5 AufenthG.

$9 \S 11$ BeschVerfV.

10 BVerwG vom 27.1.2009-1 C 40/07.

$11 \S 61$ AsylVerfG.

$12 \S 61$ Abs. 2 AsylVerfG.

13 Siehe $\S 2$ BeschVerf i.V.m. $\$ 7$ Nr. 3 BeschV.

$14 \S 58$ Abs. 1 AsylVerfG.

$15 \S 21$ Abs. 6 AufenthG.
} 
legt sie, sich mit einer kleinen Änderungsschneiderei selbständig zu machen. Die Arbeitsagentur stellt ihr die Bewilligung des Gründungszuschusses nach $\$ 57$ SGB III in Aussicht, wenn sie eine Erlaubnis zur selbständigen Tätigkeit erhält. Die Ausländerbehörde trifft auf ihren Antrag auf der Grundlage des $\mathbb{2} 21$ Abs. 6 AufenthG eine ablehnende Ermessensentscheidung, gestützt auf den Umstand, dass ihr Aufenthalt noch nicht verfestigt ist.

Neben den formalen Beschränkungen des Arbeitsmarktzugangs müssen weitere aufenthaltsrechtliche Mechanismen einbezogen werden.

Vor allem die Bindung des Aufenthalts an ein Erwerbseinkommen, welches deutlich oberhalb des soziokulturellen Existenzminimums definiert wird ${ }^{16}$, führt zu einer ständigen aufenthaltsrechtlichen Bedrohung von Migrantinnen mit befristetem Aufenthalt. Angesichts der Verweisung in den Niedriglohnsektor und der zunehmenden Einkommensabsenkungen selbst im Bereich akademischer Tätigkeiten werden die Anforderungen an das Einkommensniveau zu einer unüberwindbaren Hürde.

Die vollständige Ausblendung der gesellschaftlich unverzichtbaren Beiträge von Migrantinnen im Reproduktionsbereich wirkt sich in vielfältiger Weise diskriminierend aus.

Als Beispiel mag das jüngste Urteil des Bundesverwaltungsgerichts zum Familiennachzug dienen. ${ }^{17}$ Selbst fünf volljährige Kinder und ein Minderjähriges, welches in Deutschland geboren und zwölf Jahre hier aufgewachsen ist, führen danach nicht zu einer Genehmigung des Aufenthalts, wenn der Ehemann und Vater aus gesundheitlichen Gründen nicht in der Lage ist, ein ausreichendes Einkommen beizubringen. Eine Verletzung des Grundrechts auf Familienschutz nach Art. 6 GG sei nicht zu besorgen, da es der Familie trotz der Niederlassungserlaubnis des Vaters zuzumuten sei, ins Herkunftsland zurückzukehren („elsewhere approch“). Auch wenn die Familie sich für einen Verbleib in Deutschland entscheide, würde die Mutter für das Aufwachsen des Kindes nicht benötigt. Nicht einmal gestreift hat das Gericht dabei der Gedanke, welche Bedeutung der familiären Sozialisationsarbeit der Mutter zukommt.

Auch nach einer Trennung oder Scheidung ist das Aufenthaltsrecht der Frau wegen fehlendem oder unzureichendem Einkommen gefährdet. Zwar wird ihr ein Zeitrahmen von einem Jahr eingeräumt, in dem ihr ein Sozialleistungsbezug nicht vorgeworfen wird, danach aber gilt uneingeschränkt die Regelanforderung der "Sicherung des Lebensunterhalts“. 18 Das Bundesverwaltungsgericht betont, dass die Sicherung des Lebensunterhalts im Ausländerrecht „als eine Voraussetzung von grundlegendem staatlichen Interesse anzusehen ist" und Ausnahmen von dieser Regel grundsätzlich eng auszulegen sind. ${ }^{19}$

Da die Schonfrist von einem Jahr kaum reicht, um fehlende Deutschkenntnisse auszugleichen und zusätzlich berufliche Qualifikationen oder marktorientierte Qualifikationsanpassungen vorzunehmen, sind Frauen gezwungen, die Erzielung von Arbeitseinkommen um jeden Preis anzustreben, bei nied- rigsten Stundenlöhnen, prekären Gestaltungsformen und oftmals auch unter Vernachlässigung der Bedürfnisse der Kinder.

Typisch für diese Situation ist die Aussage einer Migrantin aus der Studie von Westphal und Behrensen:

„Also ein Jahr bevor ich fertig wurde [Erzieherinnenausbildung], hab ich mich von meinem Mann getrennt, dadurch hatte ich Schwierigkeit im Aufenthalt. Und dann bei der Ausländerbehörde meinten sie, ich hab jetzt nur noch ein Jahr, um einen festen Job zu bekommen. Dann hab ich für mich so gesagt, drei Monate, also ich gebe für mich drei Monate wo ich mich bewerbe und wenn keine Job als Erzieherin finde, dann werde ich (putzen gehen, d. V.). Obwohl ich das von Anfang an nicht wollte, weil nichts gegen Putzjob, aber ich dachte mir, ich bin noch jung, ich kann noch was anderes machen, warum soll ich das machen. Ja in diesen drei Monaten, ich hab mich zwar beworben, nur Absage obne Begründung. " (1.3., Fluchthintergrund, 1. Generation, Erzieherin) ${ }^{20}$

Als problematisch erweist sich hier auch die Neuregelung in $\mathbb{3}$ Abs. 2b SGB II, nach der alle Leistungsbezieherinnen, die nicht über das Sprachniveau B 1 des gemeinsamen Europäischen Referenzrahmens für Sprachen verfügen, zur Teilnahme an einem Integrationskurs verpflichtet werden sollen, soweit sie nicht unverzüglich in Ausbildung oder Arbeit vermittelt werden können. Diese höchst sinnvoll erscheinende Regelung beinhaltet gerade für Frauen nach einer Trennung das Risiko, in einen Integrationskurs „abgeschoben“ zu werden und dadurch ihr Aufenthaltsrecht zu riskieren. Stattdessen könnte der Spracherwerb sinnvoller mit einer Qualifizierung zur Aufnahme einer Erwerbstätigkeit verbunden werden.

\section{Ausbildungsbeihilfen}

Nicht nur die ordnungsrechtliche Genehmigung einer Erwerbstätigkeit, sondern auch die finanzielle Möglichkeit, durch Ausbildung oder Weiterbildung eine angemessene berufliche Teilhabe in dieser Gesellschaft zu erreichen, ist konstitutiv sowohl für individuelle emanzipatorische Prozesse als auch für die Verwirklichung des verfassungsrechtlich vorgegebenen Ziels einer geschlechtergerechten Gesellschaft.

Auch auf diesem Gebiet haben die Gesetzesänderungen der letzten Jahre vieles vorangebracht. War doch bis 2007 die Ausbildungsförderung für Ausländer(innen) ohne Daueraufenthalt ausschließlich von der bisher erbrachten Arbeits-

16 BVerwG vom 26.8.2008 - 1C 32.07-verlangt ein Einkommen, welches keinen ergänzenden Leistungsbezug nach SGB II mehr möglich macht.

17 BVerwG vom 30.4.2009-1C 3.08.

18 Bay. VGH vom 6.7.2009 - 19 CS 09.1365.

19 BVerwG vom 30.4.2009-1 C 3/08.

20 Westphal, Manuela/Behrensen, Birgit, Wege zum beruflichen Erfolg bei Frauen mit Migrationshintergrund der ersten und zweiten Generation und Ursachen für die gelungene Positionierung im Erwerbsleben, Osnabrück 2008, 107 (108), erhältlich im Internet: <http://www.bamf.de/nn_442266/SharedDocs/Anlagen/DE/ Migration/Publikationen/Forschung/Expertisen/ erfolgsbiographien.html> (besucht am 22. September 2009). 
leistung ihrer Eltern abhängig, wodurch junge Menschen aus Flüchtlingsfamilien, unbegleitete Minderjährige und Kinder von Erwerbsunfähigen und Arbeitslosen von qualifizierten Ausbildungen ausgeschlossen wurden. Zum 1. August 2008 erfolgte die weitgehende Öffnung der Ausbildungsbeihilfen nach BAföG und nach $\mathbb{S} 59$ ff. SGB III für Bildungsinländer(innen); in jedem Fall spätestens nach vier Jahren Aufenthalt. ${ }^{21}$ Ein echter Paradigmenwechsel wurde dann zum 1. Januar 2009 nachgeschoben, als die Ausbildungsförderung auch für Geduldete nach vier Jahren registriertem Aufenthalt in Deutschland geöffnet wurde. ${ }^{22}$ Ausgenommen bleibt unverständlicherweise die Förderung „Berufsvorbereitender Bildungsmaßnahmen“. Damit vollzog der Gesetzgeber eine entscheidende Abkehr von der bis dahin bestehenden Prämisse, nach der Ausländer(inne)n ohne Aufenthaltstitel keine auf Integration gerichteten Leistungen gewährt werden dürfen. Kennt das Grundgesetz auch kein ausdrückliches Recht auf Bildung, so lässt sich der Anspruch junger Menschen auf den Erwerb einer Berufsausbildung nicht aus dem allgemeinen Persönlichkeitsrecht nach Art. 2 Abs. 1 GG herauslösen und ausschließlich dem Deutschengrundrecht der Berufsfreiheit nach Art. 12 GG zuordnen.

Bedauerlicherweise wurde auch bei diesen Gesetzesreformen nicht auf die Beibehaltung der Hierarchisierung von Ansprüchen verzichtet, so dass es zu Differenzierungen kommt, deren gleichheitsrechtliche Legitimation zumindest fraglich ist.

Ich möchte auch das an einem Beispiel demonstrieren:

Die ukrainische Staatsangehörige heiratet einen russischen Staatsangehörigen, der seit Geburt in Deutschland lebt und eine Niederlassungserlaubnis besitzt. Nachdem sie ihre Schulausbildung (Abitur) in Kiew abgeschlossen, ein Deutschzertifikat am Goethe-Institut erworben und nachgewiesen hat, dass ihr Ehemann über 1.300 Euro Nettolohn verfügt sowie ein Visumsverfahren durchgeführt hat, darf sie schließlich nach Deutschland einreisen. Sie möchte Elektrotechnik studieren. Zunächst wird sie von ihrem Ehemann finanziert, der jedoch nach zwei Jahren arbeitslos wird. Sie kann nun Leistungen nach BAföG erhalten. Sollte sie sich von ihrem Mann trennen, werden die Leistungen weitergezahlt.

Variante:

Ihr Ehemann wird bereits kurz nach ihrer Einreise arbeitslos; um ihren Aufenthalt nicht zu gefährden, nimmt sie zunächst einen Teilzeitjob an. Nach zwei Jahren kommt es zur Trennung. Sie erhält eine Aufenthaltserlaubnis nach \31 AufenthG. Ihr Einkommen spielt dabei im ersten Jahr keine Rolle, sie kann auch Alg II beziehen. Nun möchte sie ein Studium aufnehmen. Das ist allerdings nicht mehr möglich, da sie mit der geänderten Aufenthaltserlaubnis erst nach vier Jahren einen Anspruch auf Leistungen nach BAföG hat.

Ein Studium, welches während des Zusammenlebens aufgenommen wird, erfährt eine deutliche Privilegierung gegenüber dem Studium, welches erst nach einer Trennung aufge- nommen wird. Die Differenzierung ist kaum nachvollziehbar, wird doch mit der eigenständigen Aufenthaltserlaubnis ein Verbleiberecht zugesprochen, welches in den ersten beiden Jahren des Nachzugs gerade noch fehlt. Zudem wird die getrennt lebende Frau aus aufenthaltsrechtlichen Gründen besonders dringend auf eine eigenständige Existenzsicherung angewiesen sein.

Angeführt werden könnte allenfalls, dass getrennt Lebende diese staatliche Unterstützung häufiger benötigen könnten und sie deshalb vorzugsweise auf eine schnelle Arbeitsaufnahme im Niedriglohnbereich verwiesen werden sollten. Eine im Lichte des Artikel 3 Absatz 2 GG höchst problematische Argumentation.

Zwei weitere Klippen finden sich in den Regelungen der Ausbildungsförderung nach BAföG und SGB III, die sich, obwohl generell unproblematisch, mittelbar als Hürden für Zugewanderte herausstellen können.

Zunächst werden sowohl nach $\mathbb{} 59$ SGB III als auch nach \ 7 BAföG nur Erstausbildungen gefördert. Bei der Berufsausbildungsbeihilfe werden auch nichtbetriebliche Ausbildungen als Erstausbildung berücksichtigt. Nach der Rechtsprechung des BSG kommt es nicht darauf an, ob die vorangegangene Ausbildung förderungsfähig war. ${ }^{23}$

Nach $\int 7$ Abs. 1 Satz 2 BAföG zählt auch eine im Ausland absolvierte Ausbildung, wenn sie dort zur Berufsausbildung befähigt. Die Verwaltungsvorschriften zu $\ 7$ BAföG nehmen hier zwar eine Einschränkung vor, wenn die Ausbildung im Bundesgebiet nicht anerkannt oder nicht verwertbar ist. Die Möglichkeit der Förderung einer weiteren Ausbildung bleibt allerdings auf Spätaussiedler, anerkannte Flüchtlinge und Ehegatten von Deutschen beschränkt. Ein sachlicher Grund ist hierfür nicht zu erkennen. Die Gerichte hatten zwar meist nur in Fällen von Deutschen über im Ausland (bzw. der DDR) erworbene Abschlüsse zu entscheiden. Die Argumentation stellt jedoch ohne Bezugnahme auf die Staatsangehörigkeit darauf ab, dass Ausbildungsbeihilfe wegen der besonderen Umstände des Einzelfalls ${ }^{24}$ zu bewilligen ist, wenn der im Ausland erworbene Abschluss nicht oder nur eingeschränkt genutzt werden kann, weil er nicht den tatsächlichen Anforderungen entspricht, die in der beruflichen Praxis gefordert werden. ${ }^{25}$ Das Bundesverwaltungsgericht will ein Erststudium im Ausland nur dann berücksichtigen, wenn es freiwillig gewählt wurde und zugleich auch die Möglichkeit bestanden hätte, diese Ausbildung in Deutschland durchzuführen, nur auf diesen Fall ziele $\mathbb{\int} 7$ Abs. 1 Satz 2 BAföG. ${ }^{26}$ Dieser Argumentation folgt das Verwaltungsgericht Karlsruhe ausdrücklich auch im Fall einer ausländischen Studentin. ${ }^{27}$

\footnotetext{
21 § BAföG, § 63 SGB III.

$22 \S 8$ Abs. 2a BAföG, $\S 63$ Abs. 2a SGB III.

23 BSG vom 29.1.2008 - B 7/7a AL 68/06 R.

$24 \S 7$ Abs. 2 Satz 2 BAföG.

25 OVG Hamburg vom 1.12.1993 - Bs V 123/93; BVerwG v 1.10.98, FamRZ 1999, $618 \mathrm{ff}$.

26 BVerwG v. 31.10.1996, FamRZ 1997, 847.

27 VG Karlsruhe vom 14.3.2007-10 K 1973/05.
} 
Wurde vor der Übersiedlung ein Studium im Herkunftsland begonnen, so gilt der Studienwechsel als unabweisbar, wenn die bisherigen Studienleistungen in Deutschland nicht angerechnet werden. ${ }^{28}$ Es kommt nicht darauf an, wie lange dieses Studium im Ausland bereits gedauert hat.

Eine weitere Hürde bilden die Altersgrenzen.

Für die Berufsausbildungsbeihilfe hat der Gesetzgeber in \59 SGB III keine Altersgrenze festgelegt. In den Durchführungsanweisungen der Bundesagentur für Arbeit wird jedoch eine Regelaltersgrenze bis zur Vollendung des 25. Lebensjahres festgelegt; Ausnahmen werden nicht benannt.

Nach $\mathbb{1 0}$ BAföG liegt die Altersgrenze bei 30 Jahren, wobei allerdings zahlreiche Ausnahmen vorgesehen sind, insbesondere der späte Erwerb der Zugangsberechtigung zu der gewünschten Ausbildung und die bisherige Hinderung an der Ausbildung durch persönliche Umstände. Bedauerlicherweise sind die Verwaltungsvorschriften bisher nicht an die gesetzlichen Neuregelungen in $\mathbb{\int} 8$ angepasst worden und nennen weiterhin nur Spätaussiedler, anerkannte Flüchtlinge und Ehegatten von Deutschen als Personen, die auch bei einem späten Ausbildungsbeginn gefördert werden können, wenn sich ihre Ausbildung im Herkunftsland in Deutschland nicht verwerten lässt.

Sowohl für die Berufsausbildungsbeihilfe als auch für die Leistungen nach BAföG sind untergesetzliche Lösungen zur Erleichterung des Ausbildungszugangs ohne weiteres möglich, aber auch mit Dringlichkeit einzufordern. In der Praxis zeigt sich bei später Zuwanderung, dass die schulischen und sprachlichen Voraussetzungen für eine Ausbildung erst mit einigen Jahren Verzögerung gegenüber der „Normalbiografie“ erreicht werden können. Der Ausschluss von einer qualifizierten Ausbildung steht dann oft in deutlichem Gegensatz zu den bislang bereits eingebrachten Anstrengungen und der Leistungsmotivation.

Leistungen der Ausbildungsbeihilfe sind Sozialleistungen, die nicht auf einer Beitragsleistung beruhen und führen deshalb nicht zur Sicherung des Lebensunterhalts. Die Bundesregierung sieht jedoch auch, dass junge Migrantinnen nicht einerseits durch Ausbildungshilfen zu höherwertigen Qualifizierungen motiviert werden können, wenn dadurch anderseits ihr Aufenthaltsrecht bedroht würde. Deshalb gelten nach den Verwaltungsvorschriften ${ }^{29}$ diese Leistungen nicht mehr als aufenthaltsschädlich. ${ }^{30}$

Es droht jedoch eine neue Klippe für alle Mütter:

Das Aufenthaltsgesetz verlangt im Wortlaut nur die Sicherung des eigenen Lebensunterhalts. ${ }^{31}$ Mit den geplanten Verwaltungsvorschriften soll diese Regel „gesetzes- und rechtssystematisch “ dahingehend ausgelegt werden, dass der eigene Lebensunterhalt nur als gesichert gilt, wenn auch der Lebensunterhalt der unterhaltsberechtigten Familienangehörigen gesichert ist. ${ }^{32}$ Der wiederum kann nur als gesichert gelten, wenn keine Ansprüche auf Leistungen nach SGB II oder SGB XII bestehen, unabhängig davon, ob sie tatsächlich in Anspruch genommen werden. ${ }^{33}$ Die Studentin mit Kind hat aber Leistungsansprüche auf Sozialgeld für das Kind und auf den Mehrbedarfszuschlag für sich selbst. Ihr Lebensunterhalt gilt dann, ausschließlich weil sie Mutter ist, nicht mehr als gesichert.

Auch das „Meister-BAföG“ wurde nun im Juli 2009 weitgehend für Ausländer(innen) geöffnet und in den persönlichen Leistungsvoraussetzungen den beiden anderen Ausbildungsbeihilfen angepasst. Wichtig ist, dass dabei zugleich die Anwendungsmöglichkeiten auf Bildungsmaßnahmen außerhalb der Handwerksberufe erweitert wurden und nun zum Beispiel auch Weiterbildungen im Gesundheitsbereich erfasst werden.

Wieder aber wird das Verhältnis zu im Ausland erworbenen Ausbildungen nicht ausdrücklich geregelt.

So heißt es in $\mathbb{} 2$ BAFG:

„(1) Förderfähig ist die Teilnahme an Fortbildungsmaßnahmen öffentlicher und privater Träger, die

1. einen Abschluss in einem nach $\mathbb{S} 4$ des Berufsbildungsgesetzes oder nach $\mathbb{S} 25$ der Handwerksordnung anerkannten Ausbildungsberuf, einen vergleichbaren bundes- oder landesrechtlich geregelten Berufsabschluss oder eine diesen Berufsabschlüssen entsprechende berufliche Qualifikation voraussetzen ..."

Nicht in Deutschland erworbene Abschlüsse können nur dann eine Grundlage für die Weiterbildung darstellen, wenn eine förmliche Gleichwertigkeitsfeststellung vorliegt, die für Drittstaatsangehörige nach den bisherigen Regelungen kaum zu erlangen ist. Selbst Unionsbürger(innen) könnten an dieser Hürde scheitern. Ihnen gegenüber müsste die Regelung allerdings europarechtskonform in der Weise ausgelegt werden, dass die in einem anderen EU-Staat erworbenen beruflichen Qualifikationen grundsätzlich als gleichwertig anzusehen sind.

Die Katze beißt sich aber in den Schwanz, wenn für die berufliche Ausbildung eben kein Gleichwertigkeitstestat zu erlangen ist und gerade deshalb eine Weiterbildung als Ergänzungsqualifizierung notwendig ist, um die bereits vorhandene Qualifizierung am deutschen Arbeitsmarkt verwertbar zu machen.

Eine geringfügige Ergänzung oder auch Auslegungshilfe im Rahmen von Verwaltungsvorschriften könnte hier ein hochinteressantes Feld für die mittlerweile von allen Seiten geforderte Nutzung beruflicher Abschlüsse von Migrantinnen öffnen.

Für Migrantinnen bedeutet es viel, dass sich die Möglichkeiten des Arbeitsmarktzugangs und der Ausbildungsfinanzierung in den letzten Jahren so deutlich verbessert haben. Ihre Stellung im Erwerbsleben ist das Fundament ihrer Stellung in der Gesellschaft.

Solange sich aber die deutsche Sozialgesetzgebung nicht durchringen kann, das Prinzip der Leistungshierarchisierung

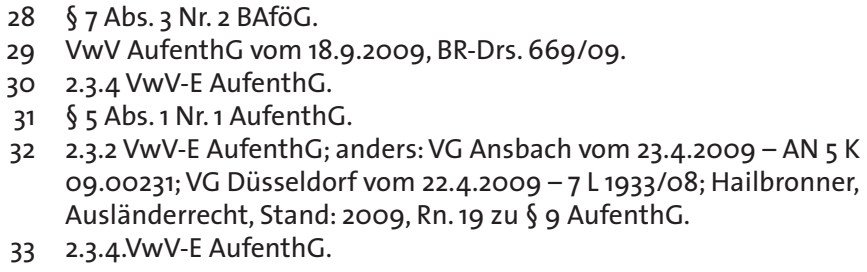


zu überwinden und klare, einfach nachzuvollziehende Teilhaberechte schafft, solange wirken Verunsicherung, Angst und das Gefühl, willkürlichen Entscheidungen ausgesetzt zu sein, der Wahrnehmung der bestehenden Möglichkeiten und Rechte entgegen.

Die angesichts der differenzierten Ausgestaltung erforderlichen Beratungsmöglichkeiten sind nicht verfügbar. Den Sozialbehörden gelingt es zunehmend weniger, ihren Beratungspflichten nach $\mathbb{S} \mathbb{S} 14,15$ SGB I nachzukommen. Eine unabhängige Beratung scheitert an viel zu wenig Anlaufstellen und auch an einer Überforderung vieler Berater(innen) angesichts der rechtlichen Komplexität. Solange diese weiter besteht, würde ich mir eine stärkere Kooperation der freien Wohlfahrtspflege mit der Anwältinnenschaft wünschen, die im Rahmen einer Betreuung nach $\mathbb{} 6$ Abs. 2 Rechtsdienstleistungsgesetz wichtige Funktionen übernehmen könnten. Dafür müssen dann aber auch die erforderlichen Gelder von der öffentlichen Hand bereitgestellt werden.

Der Einsatz für eine gleichberechtigte berufliche Teilhabe von Migrantinnen und die Anerkennung ihrer gesellschaftlich relevanten Leistungen außerhalb der Erwerbsarbeit sollte einen vollwertigen Platz in den rechtspolitischen Gleichstellungsdiskursen erhalten.

Zuzulassen, dass Migrantinnen systematisch in den Bereich unterbezahlter, prekärer persönlicher Dienstleistungen abgeschoben werden, wäre ein Verrat am Kampf der Frauenbewegung gegen die Zuweisung zu „Kinder, Küche, Kirche“.

\title{
Arbeitsmarktzugang von Migrantinnen und Migranten in der Praxis
}

\author{
Ein Erfahrungsbericht aus der Arbeit mit Migrant(inn)en in Projekten und Maßnahmen des Inter- \\ nationalen Bundes (e.V.)'
}

\author{
Anja Steuer-Loitsch \\ Programmgeschäftsführerin, Internationaler Bund, Heilbronn
}

\section{Arbeitswelt und Trends in Deutschland ${ }^{2}$}

Zentrale Thesen dieser interessanten Untersuchung sind:

- Deutschlands Bevölkerung schrumpft und wird immer älter.

- Die Bedeutung des lebenslangen Lernens steigt.

- Der Arbeitsmarkt verändert sich.

- Frauen haben zunehmende Chancen am Arbeitsmarkt.

- Der Arbeitsmarkt bietet vielfältige Möglichkeiten.

- Die Beschäftigungsprofile des Einzelnen verändert sich. Alle diese Faktoren betreffen die arbeitende Bevölkerung gleichermaßen, unabhängig von ihrer Herkunft. Da aufgrund der demografischen Entwicklung die Beschäftigungsquote von Migrant(inn)en jedoch steigen wird, sind diese Thesen im Bezug auf die Chancen und Perspektiven der Arbeitsmarktintegration dieser Bevölkerungsgruppe von besonderem Interesse. Der Bedeutungszuwachs von Wissen erhöht die Eintrittsbarriere am Arbeitsmarkt. Denn Unternehmen in Hochlohnländern sind vor allem auf ihre Innovationskraft und ihre Innovationsfähigkeit angewiesen. Auch der Umgang mit Komplexität und Veränderungsgeschwindigkeit ist für Arbeitnehmer(innen) eine sich weiter fortsetzende Herausforderung, der sie sich in ihrer Berufsbiografie stellen müssen. Konkret bedeutet dies, dass es solcher Mitarbeiter(innen) und Führungskräfte bedarf, die flexibel, mobil und anpassungsfähig sind und mit Unsicherheiten der Globalisierung (Dynamik, Schnelligkeit, Instabilität von Systemen, Unvorhersehbarkeit von Entwicklungen u.ä.m.) umgehen können.
Die Spaltung des Arbeitsmarktes (Fachkräfteengpass bei gleichzeitig hoher Arbeitslosigkeit) ist bereits heute deutlich spürbar und wird sich in Zukunft weiter verstärken. Dabei sind die Potenziale der Bevölkerung mit Migrationshintergrund gefragt.

Hier sieht das Referat für die Förderung von Integrationsprojekten für Zuwanderer ${ }^{3}$ eine Chance für Zuwander(innen) er, da diese im Zuge ihrer Integration bei uns die aus dem Herkunftsland mitgebrachten Qualifikationen, Kompetenzen und Fähigkeiten zur Anerkennung und Stärkung bringen können. Hieraus resultiert ein Ansatz des Bundesamtes für Migration und Flüchtlinge, der sogenannte „ressourcenorientierte Integrationsansatz“. Hierzu gibt es bereits zahlreiche Projekte, die die Talente und besonderen Fähigkeiten von $\mathrm{Zu}$ gewanderten aufgreifen und diesen somit zur Entfaltung verhelfen. In diesen Projekten geht es den Verantwortlichen darum, junge Frauen und Mädchen mit Migrationshintergrund zu ermutigen, die bisherigen Vorstellungen der Frauenrolle zu überdenken und die engen Grenzen nachbarschaftlicher sozialer Kontrolle zu überschreiten. Der Übergang in

1 Der Internationale Bund (IB) ist ein freier Träger der Jugend-, Sozialund Bildungsarbeit e.V. mit rund 8000 Mitarbeiter(inne)n an etwa 600 Standorten im Bundesgebiet, gegründet 1949 als Jugendsozialwerk: www.internationaler-bund.de.

2 Arbeit der Zukunft-Zukunft der Arbeit. Anforderungen und Herausforderungen. Ibe (Institut für Beschäftigung und Employability), Prof. Dr. Jutta Rump, April 2009.

3 Martin, Andreas, Mitgebrachte Kompetenzen stärken, in: Bundesamt für Migration und Flüchtlinge (Hrsg.), Blickpunkt Integration 2 (02/2009), 9, erhältlich im Internet: <http://www.soziales.sachsen. de/download/OPEN Workshop2 BAMF Anerkennung auslaendischer_Bildungsabschluesse.pdf > (besucht am 11.1.2010). 